MOHAND-OUAmar HERnANE

JEAN-LOUIS NICOLAS

\title{
Localisation de zéros de familles de trinômes
}

Annales de la faculté des sciences de Toulouse $6^{e}$ série, tome $8, \mathrm{n}^{\circ} 3$ (1999), p. 471-490

<http://www.numdam.org/item?id=AFST_1999_6_8_3_471_0>

(C) Université Paul Sabatier, 1999, tous droits réservés.

L'accès aux archives de la revue «Annales de la faculté des sciences de Toulouse » (http://picard.ups-tlse.fr/ annales/) implique l'accord avec les conditions générales d'utilisation (http://www.numdam.org/conditions). Toute utilisation commerciale ou impression systématique est constitutive d'une infraction pénale. Toute copie ou impression de ce fichier doit contenir la présente mention de copyright.

\section{Numdam}

Article numérisé dans le cadre du programme

Numérisation de documents anciens mathématiques

http://www.numdam.org/ 


\title{
Localisation de zéros de familles de trinômes ${ }^{(*)}(1)$
}

\author{
Mohand-Ouamar Hernane ${ }^{(2)}$ Et Jean-Louis Nicolas ${ }^{(3)}$
}

RÉSUMÉ. - Posons $f_{n, a}(z)=z^{n+1}-a z+a-1$ où $a$ est un nombre réel, $G_{n}(z)=(\varphi-1) z^{n+1}-\varphi z^{n}+1$ et $H_{n}(z)=\varphi z^{n+1}-(\varphi-1) z^{n}-1$ où $\varphi=(1+\sqrt{5}) / 2$. Au moyen de trois méthodes différentes, nous donnons des estimations explicites de certaines racines de ces trois familles de trinômes.

Abstract. - Let us define $f_{n, a}(z)=z^{n+1}-a z+a-1$ where $a$ is a real number, $G_{n}(z)=(\varphi-1) z^{n+1}-\varphi z^{n}+1$ and $H_{n}(z)=\varphi z^{n+1}-(\varphi-1) z^{n}-1$ where $\varphi=(1+\sqrt{5}) / 2$. We give explicit estimations of some roots of these three families of trinomials by three different methods.

\section{Introduction}

Soit $n$ un entier positif, et $a$ un nombre réel. Posons

$$
f_{n, a}(z)=z^{n+1}-a z+a-1 \text {. }
$$

La localisation des racines du trinôme $f_{n, n+1}(z)$ est étudiée dans l'article [12] (cf. aussi [9], [11] et [5]) où il est démontré que ce polynôme admet

(*) Reçu le 9 février 1999, accepté le 12 octobre 1999

(1) Recherche partiellement financée par le CNRS, Institut Girard Desargues, UPRESA 5028 et par la coopération franco-algérienne.

(2) Institut de Mathématiques, Université Houari Boumédienne, BP 32, El Alia, 16111Bab Ezzouar, Alger, Algérie. e-mail: csai@tassili.cerist.dz

(3) Institut Girard Desargues, Mathématiques, Bât. 101, Université Claude Bernard (Lyon 1), F-69622 Villeurbanne cedex, France.

e-mail: jlnicola@in2p3.fr 
$z=1$ comme racine double et également $n-1$ racines simples notées $z_{1}, z_{2}, \ldots, z_{n-1}$ et vérifiant $z_{n-k}=\overline{z_{k}}$,

$$
\frac{n}{n-1} \leqslant\left|z_{k}\right| \leqslant(2 n)^{1 / n} \quad \text { et } \quad \frac{2 k \pi}{n} \leqslant \arg z_{k} \leqslant \frac{(2 k+1) \pi}{n+1}, \quad 1 \leqslant k \leqslant \frac{n}{2} .
$$

Dans l'article [4] (cf. aussi [10]), deux autres familles de trinômes sont considérées

$$
G_{n}(z)=(\varphi-1) z^{n+1}-\varphi z^{n}+1
$$

et

$$
H_{n}(z)=\varphi z^{n+1}-(\varphi-1) z^{n}-1
$$

où $\varphi=\frac{1+\sqrt{5}}{2}$ est le nombre d'or. Notons le lien avec la famille précédente : on a $G_{n}(z)=z^{n+1} f_{n, \varphi}(1 / z)$ et $G_{n}$ est donc le polynôme réciproque de $f_{n, \varphi}$. Il est démontré dans [4] que $G_{n}$ admet $n+1$ racines simples; deux sont réelles positives, $z=1$ et une racine supérieure à 1 notée $x_{1, n}$; toutes les autres sont à l'intérieur du disque unité. Enfin, il y a une racine dans chacun des secteurs

$$
|z|<1 \text { et } \quad\left|\arg z-\frac{2 k \pi}{n}\right| \leqslant \frac{\pi}{n+1}, \quad k=1,2, \ldots, n-1 .
$$

Notons que, lorsque $n$ est pair, la racine située dans le secteur indexé par $k=n / 2$ est réelle négative; nous la désignerons par $x_{2, n}$.

Des résultats semblables sont obtenus pour la famille de trinômes (4). Il y a une seule racine réelle positive $z=1$, et une racine dans chacun des secteurs

$$
|z|<1 \quad \text { et } \quad\left|\arg z-\frac{(2 k+1) \pi}{n}\right| \leqslant \frac{\pi}{n+1}, \quad k=0,1, \ldots, n-1 .
$$

Lorsque $n$ est impair, la racine située dans le secteur indexé par $k=\frac{n-1}{2}$ est réelle négative; nous la désignerons par $x_{2, n}$, sans confusion possible avec la racine de $G_{n}$ à cause de la parité de $n$.

Dans cet article, nous nous proposons d'apporter des précisions sur les racines de ces trinômes, et ceci par trois méthodes.

La première méthode est celle des solutions approchées : il s'agit de trouver une solution approchée de l'équation, puis, à l'aide de théorèmes classiques en analyse complexe (essentiellement la formule de Taylor et le principe du maximum), prouver qu'il existe un zéro qui en soit proche. Cette méthode est utilisée dans [6], et elle a servi à démontrer le théorème 2 de [12]. Dans le paragraphe 2 ci-dessous, nous améliorons ce théorème 
2 de [12], et dans le paragraphe 3, nous appliquons la méthode des solutions approchées au trinôme (1). Voici comment, sur la suggestion de A. Schinzel, nous trouvons la solution approchée dans ce cas. On part du principe que les arguments des racines sont à peu près équidistribués, et que leurs modules sont voisins de 1 ; on fait dans (1) le changement de variables $z=r_{k} \exp \left(\frac{2 i k \pi}{n+1}+i \psi_{k}\right)$. En séparant partie réelle et partie imaginaire, on obtient

$$
r_{k}^{n+1} \cos \left((n+1) \psi_{k}\right)=a r_{k} \cos \left(\frac{2 k \pi}{n+1}+\psi_{k}\right)+a-1
$$

et

$$
r_{k}^{n+1} \sin \left((n+1) \psi_{k}\right)=a r_{k} \sin \left(\frac{2 k \pi}{n+1}+\psi_{k}\right) .
$$

En ajoutant les carrés de (5) et (6), il vient

$$
r_{k}^{2(n+1)}=a^{2} r_{k}^{2}-2 a(a-1) r_{k} \cos \left(\frac{2 k \pi}{n+1}+\psi_{k}\right)+(a-1)^{2} .
$$

Dans le membre de droite de (7), on substitue $\psi_{k}=0$ et $r_{k}=1$. Cela donne

$$
r_{k}^{2(n+1)}=a^{2}-2 a(a-1) \cos \left(\frac{2 k \pi}{n+1}\right)+(a-1)^{2}=1+4 a(a-1) \sin ^{2} \frac{k \pi}{n+1}
$$

d'où l'on déduit la valeur de $r_{k}$ donnée par (24) ci-dessous. Ensuite, dans (6), on substitue $r_{k}=1$, on assimile $\sin \left(\frac{2 k \pi}{n+1}+\psi_{k}\right)$ à $\frac{2 k \pi}{n+1}$ et $\sin \left((n+1) \psi_{k}\right)$ à $(n+1) \psi_{k}$, et cela fournit la valeur de $\psi_{k}$ donnée dans (25).

La deuxième méthode utilise le théorème des fonctions implicites analytiques. Dans [12], le théorème 4 donne pour $\left|z_{1}\right|$ et $\arg z_{1}$ (où $z_{1}$ est la racine de $f_{n, n+1}$ définie par (2)) un développement limité suivant les puissances de $1 / n$. H. Delange a observé que l'on pouvait, de façon à la fois plus élégante et plus précise, obtenir pour $z_{1}$ un développement en série entière par rapport à $1 / n$, en utilisant le théorème des fonctions implicites. De plus, T. Fack nous a signalé une version explicite du théorème des fonctions implicites utilisant le rectangle de sécurité de l'équation différentielle associée. Tout ceci est développé dans le paragraphe 4. Dans [4], on donne pour le zéro réel négatif $x_{2, n}$ de $H_{n}$ ou $G_{n}$ l'estimation

$$
x_{2, n}=-1+(1+o(1)) \frac{\log 5}{2 n}, \quad n \rightarrow \infty .
$$

La méthode du théorème des fonctions implicites analytiques donne pour $x_{2, n}$ (en distinguant les cas $n$ impair et $n$ pair) un développement en série 
entière suivant les puissances de $1 / n$ et convergent pour $n$ assez grand. Ceci fera l'objet du paragraphe 5 .

La troisième méthode donne un développement en série hypergéométrique des racines d'un trinôme. Elle sera exposée dans le paragraphe 6, où la proposition 2 reprend la formulation de Belardinelli (cf. [1]) pour la racine de l'équation $y^{N}+x y^{P}-1=0$ telle que $y(0)=1$. La référence [1] nous a été communiquée par P. Barrucand. G. Belardinelli donne deux démonstrations de son résultat; l'une utilise la transformée de Mellin, tandis que l'autre utilise le théorème d'inversion de Lagrange. Nous en donnerons, à l'aide de la formule d'inversion de Lagrange, une preuve courte qui nous a été fournie par J. Zeng. Dans l'article [4], on donne pour $x_{1, n}$, le zéro positif différent de 1 de $G_{n}(z)$, l'estimation

$$
\varphi^{2}\left(1-\varphi^{-2 n}\right) \leqslant x_{1, n} \leqslant \varphi^{2}\left(1-\varphi^{-2 n-1}\right) .
$$

Dans le théorème 5 , nous améliorons nettement cette estimation en donnant pour $x_{1, n}$ un développement en série hypergéométrique très rapidement convergent.

Nous avons plaisir à remercier chaleureusement les mathématiciens qui nous ont aidé dans l'élaboration de cet article et que nous avons cité ou que nous citerons en précisant la nature de leur aide : P. Barrucand, $H$. Delange, J.-P. Demailly, B. Salvy, A. Schinzel, T. Fack, J. Zeng, ainsi que K. Dilcher et K.B. Stolarsky pour nous avoir communiqué leurs articles.

\section{Solutions approchées : $z^{n+1}-(n+1) z+n=0$}

J.-L. Nicolas et A. Schinzel montrent dans [12] (cf. aussi [9]) que le théorème 1 ci-dessous est vérifié pour la constante $A=10^{40}$. Dans ce qui suit, nous abaissons sensiblement cette constante.

THÉORÈME 1. - Il existe une constante absolue $A \geqslant 1(A=14$ convient) avec la propriété : pour tout $n \geqslant 2 A$ et pour tout entier $k$ vérifiant $A \leqslant k \leqslant n-A$, si l'on pose

$$
r_{k}=\left(2(n+1) \sin \left(\frac{4 k+1}{4 n+2} \pi\right)\right)^{\frac{1}{n+1}} \quad \text { et } \quad \rho=3 \frac{r_{k}-1}{r_{k}^{n}-1}
$$

alors

(i) $r_{k}>1$ et $\rho \leqslant 3 / n$.

(ii) L'équation $f(z)=f_{n, n+1}(z)=z^{n+1}-(n+1) z+n=0$ admet une 
racine $z_{k}$ satisfaisant à l'inégalité

$$
\left|z_{k}-x_{k}\right| \leqslant \rho \quad \text { avec } \quad x_{k}=r_{k} \exp \left(i \pi \frac{4 k+1}{2 n+1}\right) .
$$

DÉmonstration DU THÉorème 1 (i). - Pour tout $k$ vérifiant $A \leqslant$ $k \leqslant n+1-A$, on a $\frac{4 k+1}{4 n+2} \pi \in[0, \pi]$; donc, en utilisant l'inégalité $\sin (\pi t) \geqslant$ $2 \min (t, 1-t)$ valable pour $0 \leqslant t \leqslant 1$, on a

$$
r_{k}^{n+1}=2(n+1) \sin \left(\frac{4 k+1}{4 n+2} \pi\right) \geqslant \frac{4 n+4}{4 n+2}(4 \min (k, n-k)+1)>4 A+1 .
$$

Comme $A \geqslant 1$, il en résulte que $r_{k}>1$ et puisque $n \geqslant 2 A$

$$
r_{k}^{n}>(4 A+1)^{\frac{n}{n+1}} \geqslant R(A) \text { avec } R(A)=(4 A+1)^{\frac{2 A}{2 A+1}} .
$$

Notons que la fonction $t \mapsto R(t)$ est croissante pour $t>0$. Grace à l'inégalité entre les moyennes arithmétiques et géométriques, on obtient la majoration

$$
\rho=3 \frac{r_{k}-1}{r_{k}^{n}-1}=\frac{3}{1+r_{k}+\ldots+r_{k}^{n-1}} \leqslant \frac{3}{n r_{k}^{(n-1) / 2}} \leqslant \frac{3}{n} .
$$

Mais on peut majorer $\rho$ encore mieux. En utilisant l'inégalité $t-1 \leqslant t \log t$, valable pour tout $t \geqslant 1$, et (12), il vient:

$$
\rho \leqslant \frac{3 r_{k} \log r_{k}}{r_{k}^{n}-1}=\frac{3 r_{k}}{n} w\left(r_{k}^{n}\right) \leqslant \frac{3 r_{k}}{n} w(R(A))
$$

en posant

$$
w(t)=\frac{\log t}{t-1}
$$

et en notant que $w(t)$ est une fonction décroissante en $t$ pour $t>1$.

Lemme 1. - Avec les notations du théorème 1, on a

(i) $\left|f\left(x_{k}\right)\right| \leqslant(n+1)\left(r_{k}-1\right)+1$

(ii) $\left|f^{\prime}\left(x_{k}\right)\right| \geqslant(n+1)\left(r_{k}^{n}-1\right)=\frac{3}{\rho}(n+1)\left(r_{k}-1\right)$

(iii) Pour $j \geqslant 2,\left|f^{(j)}\left(x_{k}\right)\right|=j !\left(\begin{array}{c}n+1 \\ j\end{array}\right) r_{k}^{n+1-j}$.

DÉmonstration. - Le lemme 1 est démontré dans [12], p. 169.

Lemme 2.-Avec les notations du théorème 1, on a

$$
S=\sum_{j=2}^{n+1} \frac{\left|f^{(j)}\left(x_{k}\right)\right|}{j !} \rho^{j} \leqslant \frac{9}{2}\left(1+\frac{1}{2 A}\right) \exp \left(\frac{\log (4 A+2)}{2 A+1}\right) \frac{y(R(A))}{1-w(R(A))}
$$


où $R(A)$ et $w(t)$ sont définis par (12) et (15) et

$$
y(x)=\frac{x \log ^{2} x}{(x-1)^{2}}=\left(\frac{\log (\sqrt{x})}{\sinh (\log \sqrt{x})}\right)^{2}
$$

est une fonction décroissante de $x$ pour $x \geqslant 1$.

DÉMONSTRATION DU LEMME 2. - La fonction $t \mapsto \sinh t / t$ est analytique et les coefficients dans son développement de Mac Laurin sont positifs. Elle est donc croissante pour $t \geqslant 0$, et cela entraîne la décroissance de $y(x)$ pour $x \geqslant 1$.

En utilisant le lemme 1, il vient

$$
\begin{aligned}
S & \leqslant \sum_{j=2}^{n+1}\left(\begin{array}{c}
n+1 \\
j
\end{array}\right) \rho^{j} r_{k}^{n+1-j} \\
& =\frac{n(n+1)}{2} \rho^{2} r_{k}^{n-1}\left(1+\frac{n-1}{3} \frac{\rho}{r_{k}}+\frac{(n-1)(n-2)}{3 \cdot 4}\left(\frac{\rho}{r_{k}}\right)^{2}+\ldots\right) \\
& \leqslant \frac{n(n+1)}{2} \rho^{2} r_{k}^{n-1} \frac{1}{1-\frac{n}{3} \frac{\rho}{r_{k}}} \leqslant \frac{n(n+1)}{2} \rho^{2} r_{k}^{n-1} \frac{1}{1-w(R(A))}
\end{aligned}
$$

en utilisant (14). On observe ensuite que $\frac{n+1}{n} \leqslant 1+\frac{1}{2 A}$ (puisque $n \geqslant 2 A$ ) et cela donne

$$
S \leqslant \frac{n^{2}}{2}\left(1+\frac{1}{2 A}\right) \rho^{2} r_{k}^{n-1} \frac{1}{1-w(R(A))} .
$$

Il reste à majorer $\rho^{2} r_{k}^{n-1}$. Par (14), (16) et (12), on a

$$
\rho^{2} r_{k}^{n-1} \leqslant \frac{9 r_{k}^{n+1} \log ^{2} r_{k}}{\left(r_{k}^{n}-1\right)^{2}}=\frac{9 r_{k}}{n^{2}} y\left(r_{k}^{n}\right) \leqslant \frac{9 r_{k}}{n^{2}} y(R(A)) .
$$

Enfin, par (9),

$$
r_{k} \leqslant(2(n+1))^{\frac{1}{n+1}}=\exp \left(\frac{\log (2 n+2)}{n+1}\right) \leqslant \exp \left(\frac{\log (4 A+2)}{2 A+1}\right)
$$

puisque $n \geqslant 2 A$ et que la fonction $t \mapsto \frac{\log (2 t+2)}{t+1}$ est décroissante pour $t \geqslant \frac{e}{2}-1$. Le lemme 2 découle alors de (17), (18) et (19).

DÉmonstration DU THÉORÈme 1 (ii). - Supposons que $f$ ne s'annule pas dans le disque de centre $x_{k}$ et de rayon $\rho$; on pourrait appliquer dans ce disque le principe du maximum à la fonction $1 / f:$ il existerait $z$, avec 
$\left|z-x_{k}\right|=\rho$ tel que $\left|\frac{1}{f\left(x_{k}\right)}\right| \leqslant\left|\frac{1}{f(z)}\right|$. Pour démontrer (ii), nous allons montrer que pour tout $z$, tel que $\left|z-x_{k}\right|=\rho$, on a

$$
|f(z)|>\left|f\left(x_{k}\right)\right| .
$$

La formule de Taylor s'écrit :

$$
f(z)=f\left(x_{k}\right)+\left(z-x_{k}\right) f^{\prime}\left(x_{k}\right)+\sum_{j=2}^{n+1} \frac{f^{(j)}\left(x_{k}\right)}{j !}\left(z-x_{k}\right)^{j}
$$

et par suite on a

$$
|f(z)| \geqslant\left|f^{\prime}\left(x_{k}\right)\right| \rho-\left|f\left(x_{k}\right)\right|-\sum_{j=2}^{n+1} \frac{\left|f^{(j)}\left(x_{k}\right)\right|}{j !} \rho^{j} .
$$

Le lemme 1 permet d'écrire :

$$
\begin{aligned}
\left|f^{\prime}\left(x_{k}\right)\right| \rho-2\left|f\left(x_{k}\right)\right| & \geqslant 3(n+1)\left(r_{k}-1\right)-2(n+1)\left(r_{k}-1\right)-2 \\
& =(n+1)\left(r_{k}-1\right)-2 \\
& \geqslant(n+1) \log r_{k}-2=\log r_{k}^{n+1}-2 \\
& \geqslant \log (4 A+1)-2
\end{aligned}
$$

par (11). De (21) et du lemme 2, on déduit alors

$$
\begin{gathered}
|f(z)|-\left|f\left(x_{k}\right)\right| \geqslant\left|f^{\prime}\left(x_{k}\right)\right| \rho-2\left|f\left(x_{k}\right)\right|-\sum_{j=2}^{n+1} \frac{\left|f^{(j)}\left(x_{k}\right)\right|}{j !} \rho^{j} \\
\geqslant \log (4 A+1)-2-\frac{9}{2}\left(1+\frac{1}{2 A}\right) \exp \left(\frac{\log (4 A+2)}{2 A+1}\right) \frac{y(R(A))}{1-w(R(A))} .
\end{gathered}
$$

Mais le membre de droite de cette inégalité est une fonction croissante de $A$, pour $A \geqslant 1$, car $R$ est croissante, et $y$ et $w$ sont décroissantes, ainsi que les fonctions $1+\frac{1}{2 A}$ et $\frac{\log (4 A+2)}{2 A+1}$. Pour $A=14$, il vaut $0.1769>0$, cela prouve (20) et achève la preuve du théorème 1 .

Remarque. - Les calculs numériques effectués pour $n \leqslant 200$ montrent que (10) est vérifiée pour tout $k$ tel que $0 \leqslant k \leqslant n$. Le théorème 1 est donc vraisemblablement exact pour tout $k, 0 \leqslant k \leqslant n$, mais nous n'avons pas réussi à le prouver sans la restriction $A \leqslant k \leqslant n-A$. Dans la définition de $\rho$ dans (9), le 3 est arbitraire (il doit cependant être supérieur à 2). En l'augmentant, on pourrait abaisser la valeur de $A$ dans le théorème 1. Pour les petites valeurs de $k$, le théorème 3 exposé dans le paragraphe 4 fournira une meilleure approximation des racines. 


\section{Solutions approchées : $z^{n+1}-a z+a-1=0$}

THÉORÈme 2 . - Soit a un nombre réel, $a \geqslant 1$. Pour $n \geqslant 1$, on définit l'équation trinôme

$$
f(z)=f_{n, a}(z)=z^{n+1}-a z+a-1=0 .
$$

Pour $1 \leqslant k \leqslant n+1$, on pose

$$
x_{k}=r_{k} \exp \left(i\left(\frac{2 k \pi}{n+1}+\psi_{k}\right)\right)
$$

avec

$$
r_{k}=\left(1+4 a(a-1) \sin ^{2} \frac{k \pi}{n+1}\right)^{\frac{1}{2(n+1)}}
$$

et

$$
\psi_{k}=\frac{2 k \pi a}{(n+1)^{2}} .
$$

Soient trois nombres réels $\alpha, \beta, \gamma$, vérifiant $0<\beta \leqslant \gamma, \alpha>0$ et

$$
\alpha(1-\beta) \geqslant \gamma(84 \gamma+13 \beta+44 \beta \gamma)+\alpha^{2} e^{\alpha}\left(1 / 2+10 \gamma^{2}\right) .
$$

On suppose que

$$
n \geqslant 9, \quad \frac{a}{n+1} \leqslant \beta \quad \text { et } 1 \leqslant k \leqslant \gamma \frac{n+1}{a} .
$$

Alors, l'équation (22) admet une racine dans le disque de centre $x_{k}$ et de rayon $\rho=\alpha /(n+1)$.

Dans (26), on peut prendre par exemple $\alpha=0.7, \beta=0.1, \gamma=0.02$.

Lorsque $a=\varphi=\frac{1+\sqrt{5}}{2}, k=1$ et $n \geqslant 99$, on peut prendre $\alpha=\frac{300}{(n+1)^{2}}$, $\beta=\gamma=\frac{\varphi}{n+1}$.

DÉMONSTRATION. - Montrons d'abord ce dernier point. Lorsque $a=\varphi$ et $n \geqslant 99$, on a

$$
\alpha=\frac{300}{(n+1)^{2}} \leqslant \frac{3}{n+1} \leqslant \frac{3}{100} \quad \text { et } \quad \beta=\gamma=\frac{\varphi}{n+1} \leqslant \frac{2}{n+1} \leqslant \frac{2}{100} .
$$

Il s'ensuit que

$$
\begin{gathered}
\alpha(1-\beta) \geqslant \alpha\left(1-\frac{2}{100}\right)=\frac{294}{(n+1)^{2}} \\
-478-
\end{gathered}
$$


Localisation de zéros de familles de trinômes

$$
\begin{aligned}
\gamma(84 \gamma+13 \beta+44 \beta \gamma) & =\beta^{2}(84+13+44 \beta) \leqslant \beta^{2}\left(97+\frac{88}{100}\right) \\
& \leqslant 98 \frac{\varphi^{2}}{(n+1)^{2}} \leqslant \frac{257}{(n+1)^{2}} \\
\alpha^{2} e^{\alpha}\left(1 / 2+10 \gamma^{2}\right) & \leqslant \frac{300}{(n+1)^{2}} \frac{3}{100} e^{3 / 100}\left(\frac{1}{2}+10 \frac{4}{10000}\right) \leqslant \frac{5}{(n+1)^{2}},
\end{aligned}
$$

ce qui prouve (26).

Ensuite, nous suivrons le même plan que dans la démonstration du théorème 1 : avant d'appliquer la formule de Taylor, il nous faut majorer $\left|f\left(x_{k}\right)\right|$, minorer $\left|f^{\prime}\left(x_{k}\right)\right|$ et majorer le terme de reste.

En appliquant à (24) l'inégalité $(1+t)^{\lambda} \leqslant 1+\lambda t$, valable pour $t \geqslant 0$ et $0 \leqslant \lambda \leqslant 1$ et l'inégalité $\sin t \leqslant t$ valable pour $t \geqslant 0$, on obtient

$$
1 \leqslant r_{k}^{n+1} \leqslant 1+\frac{2 a(a-1) \pi^{2} k^{2}}{(n+1)^{2}} \text { et } 1 \leqslant r_{k} \leqslant 1+\frac{2 a(a-1) \pi^{2} k^{2}}{(n+1)^{3}} .
$$

Nous utiliserons aussi l'inégalité bien connue des probabilistes (cf. [7], p. 512)

$$
\left|e^{i t}-1-i t\right| \leqslant \frac{t^{2}}{2}, \quad t \in \mathbb{R}
$$

qui peut se démontrer en appliquant la formule de Taylor avec reste intégral : en posant $w(t)=e^{i t}$, on a $\left|e^{i t}-1-i t\right|=\left|\int_{0}^{t}(t-u) w^{\prime \prime}(u) d u\right| \leqslant \int_{0}^{t}(t-u)\left|w^{\prime \prime}(u)\right| d u=\int_{0}^{t}(t-u) d u=\frac{t^{2}}{2}$. Il vient ensuite par (22), (23), (24) et (25)

$$
\begin{gathered}
f\left(x_{k}\right)=r_{k}^{n+1} e^{(n+1) \psi_{k} i}-a r_{k} e^{\frac{2 i \pi k}{n+1}+i \psi_{k}}+a-1 \\
=\left(e^{(n+1) \psi_{k} i}-1-i(n+1) \psi_{k}\right)-a\left(e^{\frac{2 i \pi k}{n+1}+i \psi_{k}}-1-\frac{2 i \pi k}{n+1}-i \psi_{k}\right) \\
-i a \psi_{k}+\left(r_{k}^{n+1}-1\right) e^{(n+1) \psi_{k} i}-a\left(r_{k}-1\right) e^{\frac{2 i \pi k}{n+1}+i \psi_{k}} .
\end{gathered}
$$

En utilisant deux fois la majoration (29), on obtient

$$
\left|f\left(x_{k}\right)\right| \leqslant \frac{(n+1)^{2} \psi_{k}^{2}}{2}+\frac{a}{2}\left(\frac{2 \pi k}{n+1}+\psi_{k}\right)^{2}+a \psi_{k}+\left(r_{k}^{n+1}-1\right)+a\left(r_{k}-1\right),
$$

et par (25) et (28), il suit

$$
\left|f\left(x_{k}\right)\right| \leqslant \frac{2 k^{2} \pi^{2} a^{2}}{(n+1)^{2}}+\frac{2 a \pi^{2} k^{2}}{(n+1)^{2}}\left(1+\frac{a}{n+1}\right)^{2}+\frac{2 a^{2} k \pi}{(n+1)^{2}}
$$




$$
+\frac{2 a(a-1) k^{2} \pi^{2}}{(n+1)^{2}}+\frac{2 a^{2}(a-1) k^{2} \pi^{2}}{(n+1)^{3}} .
$$

Après réduction des termes semblables, le membre de droite ci-dessus s'écrit

$$
\frac{2 a^{2} k^{2} \pi^{2}}{(n+1)^{2}}\left(2+\frac{a}{n+1}\left(1+\frac{1}{n+1}\right)+\frac{1}{n+1}+\frac{1}{k \pi}\right) .
$$

En tenant compte de l'hypothèse $n \geqslant 9$, on majore $\frac{1}{n+1}$ par $\frac{1}{10}, \pi^{2}$ par 10 et $2 \pi$ par 6.5 , et l'on obtient

$$
\left|f\left(x_{k}\right)\right| \leqslant \frac{a^{2} k^{2}}{(n+1)^{2}}\left(42+22 \frac{a}{n+1}\right)+6.5 \frac{a^{2} k}{(n+1)^{2}} .
$$

Compte tenu des hypothèses (27), on en déduit

$$
\left|f\left(x_{k}\right)\right| \leqslant \gamma^{2}(42+22 \beta)+6.5 \beta \gamma=\gamma(42 \gamma+22 \beta \gamma+6.5 \beta) .
$$

Minorons maintenant la dérivée : on a, par (22), (23) et (24)

$$
\left|f^{\prime}\left(x_{k}\right)\right|=\left|(n+1) x_{k}^{n}-a\right| \geqslant(n+1)\left|x_{k}^{n}\right|-a=(n+1) r_{k}^{n}-a \geqslant n+1-a,
$$

et sous les hypothèses (27), on a

$$
\left|f^{\prime}\left(x_{k}\right)\right| \geqslant(n+1)\left(1-\frac{a}{n+1}\right) \geqslant(n+1)(1-\beta) .
$$

Enfin, soit $z \in \mathbb{C}$ avec $\left|z-x_{k}\right|=\rho=\alpha /(n+1)$, Il vient

$$
\int_{x_{k}}^{z}(z-t) f^{\prime \prime}(t) d t=n(n+1) \int_{x_{k}}^{z} t^{n-1}(z-t) d t .
$$

Par le changement de variables $t=x_{k}+u\left(z-x_{k}\right)$, cette intégrale devient

$$
\int_{x_{k}}^{z}(z-t) f^{\prime \prime}(t) d t=n(n+1) \int_{0}^{1}\left(x_{k}+u\left(z-x_{k}\right)\right)^{n-1}(1-u)\left(z-x_{k}\right)^{2} d u
$$

et on peut écrire

$$
\begin{aligned}
\left|\int_{x_{k}}^{z}(z-t) f^{\prime \prime}(t) d t\right| & \leqslant n(n+1) \rho^{2}\left(r_{k}+\rho\right)^{n-1} \int_{0}^{1}(1-u) d u \\
& =\frac{n(n+1)}{2} \rho^{2}\left(r_{k}+\rho\right)^{n-1} \\
& \leqslant \frac{(n+1)^{2}}{2} \rho^{2} r_{k}^{n-1}\left(1+\frac{\rho}{r_{k}}\right)^{n-1} \\
& \leqslant \frac{(n+1)^{2}}{2} \rho^{2} r_{k}^{n+1}(1+\rho)^{n+1} .
\end{aligned}
$$


Comme $\rho=\alpha /(n+1)$, on a, par (28)

$$
\left|\int_{x_{k}}^{z}(z-t) f^{\prime \prime}(t) d t\right| \leqslant \frac{\alpha^{2}}{2} \exp (\alpha)\left(1+\frac{2 a^{2} k^{2} \pi^{2}}{(n+1)^{2}}\right)
$$

et compte tenu des hypothèses (27),

$$
\left|\int_{x_{k}}^{z}(z-t) f^{\prime \prime}(t) d t\right| \leqslant \frac{\alpha^{2}}{2} \exp (\alpha)\left(1+20 \gamma^{2}\right) .
$$

Supposons que $f$ ne s'annule pas dans le disque de centre $x_{k}$ et de rayon $\rho$; on pourrait appliquer dans ce disque le principe du maximum à la fonction $1 / f$ : il existerait $z$, avec $\left|z-x_{k}\right|=\rho$ tel que $\left|\frac{1}{f\left(x_{k}\right)}\right| \leqslant\left|\frac{1}{f(z)}\right|$. Pour démontrer le théorème 2 , nous allons montrer que pour tout $z$, tel que $\left|z-x_{k}\right|=\rho$, on a

$$
|f(z)|>\left|f\left(x_{k}\right)\right| .
$$

La formule de Taylor avec reste intégral s'écrit :

$$
f(z)=f\left(x_{k}\right)+\left(z-x_{k}\right) f^{\prime}\left(x_{k}\right)+\int_{x_{k}}^{z}(z-t) f^{\prime \prime}(t) d t
$$

d'où

$$
|f(z)| \geqslant\left|\left(z-x_{k}\right) f^{\prime}\left(x_{k}\right)\right|-\left|f\left(x_{k}\right)\right|-\left|\int_{x_{k}}^{z}(z-t) f^{\prime \prime}(t) d t\right| .
$$

L'inégalité (36) résultera donc de

$$
\left.\mid\left(z-x_{k}\right) f^{\prime}\left(x_{k}\right)\right)|=\rho| f^{\prime}\left(x_{k}\right)|>2| f\left(x_{k}\right)|+| \int_{x_{k}}^{z}(z-t) f^{\prime \prime}(t) d t \mid .
$$

$\operatorname{Par}(33), \rho\left|f^{\prime}\left(x_{k}\right)\right|>\alpha(1-\beta)$, et (37) résulte de (32), (35) et (26).

\section{Le théorème des fonctions implicites}

On trouvera un énoncé du théorème des fonctions implicites analytiques dans [2], 7.8. Nous présentons ci-dessous une version effective, communiquée par T. Fack.

Proposition 1. - Soit $F(x, y)$ une fonction de deux variables complexes, analytique dans un ouvert $\Omega \subset \mathbb{C}^{2}$ contenant le rectangle $\mathcal{R}=$ $\mathcal{R}\left(x_{0}, y_{0}, s, r\right)=\left\{(x, y) \in \mathbb{C}^{2},\left|x-x_{0}\right| \leqslant s,\left|y-y_{0}\right| \leqslant r\right\}$. On suppose que $F\left(x_{0}, y_{0}\right)=0$, que $\frac{\partial F}{\partial y}(x, y) \neq 0$ pour tout point $(x, y) \in \mathcal{R}$ et que, de 
plus, le rectangle $\mathcal{R}$ est un rectangle de sécurité pour la fonction $G(x, y)=$ $-\frac{\partial F}{\partial x}(x, y) / \frac{\partial F}{\partial y}(x, y), c^{\prime} e s t-\grave{a}$-dire

$$
\left|x-x_{0}\right| \leqslant s \quad \text { et } \quad\left|y-y_{0}\right| \leqslant r \quad \Longrightarrow \quad|G(x, y)|<M=r / s .
$$

Alors, il existe une fonction $y=y(x)$ définie dans le disque $\left|x-x_{0}\right| \leqslant s$ telle que $F(x, y(x))=0$ et $y\left(x_{0}\right)=y_{0}$. Dans ce disque, cette fonction est analytique et vérifie $\left|y(x)-y_{0}\right| \leqslant r$.

DÉMONSTRATION. - On sait que l'équation différentielle $y^{\prime}=G(x, y)$ admet une solution $y(x)$ analytique dans le disque $\left|x-x_{0}\right| \leqslant s$ vérifiant $y\left(x_{0}\right)=y_{0}$ et $\left|y(x)-y_{0}\right| \leqslant r$ (cf. par exemple [3], p. 124 qui traite le cas réel, mais, comme nous l'a confirmé J.-P. Demailly, le cas complexe se traiterait de la même façon). La fonction de $x, F(x, y(x))$ a pour dérivée $\frac{\partial F}{\partial x}(x, y(x))+$ $y^{\prime}(x) \frac{\partial F}{\partial y}(x, y(x))=0$, elle est donc constante et vaut $F\left(x_{0}, y_{0}\right)=0$.

LEMME 3. - L'équation complexe $e^{z}=1+z$ admet la racine $z_{0}=0$ et pour chaque valeur de l'entier $k \geqslant 1$, admet pour racine le nombre $z_{k}=$ $x_{k}+i y_{k}$ avec $2 k \pi<y_{k}<2 k \pi+\pi / 2$ et $x_{k}>1$ et son conjugué $\overline{z_{k}}$. Ce sont les seules racines de cette équation. On a

$$
z_{1} \approx 2.08+7.46 i, \quad z_{2} \approx 2.66+13.88 i, \quad z_{3} \approx 3.02+20.22 i \text {. }
$$

DÉMONSTRATION. - On pose $z=x+i y$. La seule racine réelle est $z=0$, et $\mathrm{si} z$ est racine, $\bar{z}$ est aussi racine. On peut donc supposer $y>0$. De plus, la relation

$$
e^{x}(\cos y+i \sin y)=1+x+i y
$$

montre que si $z$ est racine, $y$ n'est pas un multiple de $\pi$ et $x \neq-1$. En comparant les modules et les arguments, on obtient

$$
e^{x}=\sqrt{(1+x)^{2}+y^{2}}
$$

et

$$
1+x=y \operatorname{cotg} y .
$$

En éliminant $x$, on obtient

$$
H(y):=y \operatorname{cotg} y-1-\log y+\log |\sin y|=0 .
$$

La dérivée vaut

$$
H^{\prime}(y)=-\frac{(y \operatorname{cotg} y-1)^{2}+y^{2}}{y}<0 .
$$


Sur chaque intervalle ouvert $] k \pi,(k+1) \pi[$, la fonction $H$ est continue et décroissante. On a $\lim _{y \rightarrow 0} H(y)=0$ et il n'y a donc pas de racines dans l'intervalle $] 0, \pi$ [. On suppose $y>\pi$. La relation (38) implique $e^{2 x} \geqslant y^{2}>\pi^{2}$ et donc $x>1$. Sur les intervalles $] 2 k \pi-\pi / 2,2 k \pi[, \operatorname{cotg} y<0$, ce qui est impossible par (39). Enfin, on a $\lim _{y \rightarrow 2 k \pi, y>2 k \pi} H(y)=+\infty$ et $H(2 k \pi+\pi / 2)<0$ et cela achève la preuve du lemme 3 .

THÉORÈME 3. - Soit $f(z)=f_{n, n+1}(z)=z^{n+1}-(n+1) z+n$, et soit $b$ une racine complexe non nulle de l'équation $e^{t}=1+t$. Il existe des coefficients complexes $c_{m}, m=1,2, \ldots$, tels que, pourn suffisamment grand, la série $1+\sum_{m=1}^{\infty} c_{m} n^{-m}$ soit convergente de somme $S_{n}$, et $S_{n}$ est racine de l'équation $f(z)=0$. On a

$$
\begin{gathered}
c_{1}=b, c_{2}=\frac{b(b-1)}{2}, c_{3}=\frac{b\left(4 b^{2}-11 b+9\right)}{24}, c_{4}=\frac{b\left(2 b^{3}-10 b^{2}+21 b-15\right)}{48}, \\
c_{5}=\frac{b\left(48 b^{4}-362 b^{3}+1330 b^{2}-2445 b+1575\right)}{5760}
\end{gathered}
$$

De plus, lorsque $b=2.08 \ldots+i 7.46 \ldots$, les coefficients $c_{m}$ sont majorés en module par $1.4 \times 25^{m}$.

DÉmonstration. - Dans l'équation $f(z)=0$, faisons les changements de variables $z=\exp (x y)$ et $n=1 / x$. On obtient:

$$
e^{y} e^{x y}-\left(\frac{1}{x}+1\right) e^{x y}+\frac{1}{x}=0
$$

qui peut se mettre sous la forme

$$
\frac{e^{y}-1}{y}-1=\frac{e^{-x y}-1}{-x y}-1
$$

ou encore $F(x, y)=0$ avec

$$
F(x, y)=g(y)-g(-x y) \quad \text { et } \quad g(t)=\frac{e^{t}-1-t}{t} .
$$

La fonction $F(x, y)$ est analytique dans $\mathbb{C}^{2}$. On a $F(0, b)=0$ et comme

$$
\frac{\partial F}{\partial y}(x, y)=g^{\prime}(y)+x g^{\prime}(-x y)=\frac{(y-1) e^{y}+1}{y^{2}}+x \frac{1-(x y+1) e^{-x y}}{x^{2} y^{2}}
$$

on a

$$
\frac{\partial F}{\partial y}(0, b)=\frac{(b-1) e^{b}+1}{b^{2}}=\frac{(b-1)(b+1)+1}{b^{2}}=1 \neq 0 .
$$


Avec les notations de la proposition 1 , il existe donc un rectangle $\mathcal{R}_{1}=$ $\mathcal{R}\left(0, b, s_{1}, r\right)$ dans lequel la dérivée partielle $\frac{\partial F}{\partial y}(x, y)$ ne s'annule pas. La fonction $G(x, y)=-\frac{\partial F}{\partial x}(x, y) / \frac{\partial F}{\partial y}(x, y)$ est majorée en module dans $\mathcal{R}_{1}$ par, disons, $M$ et en posant $s=\min \left(s_{1}, r / M\right)$, le rectangle $\mathcal{R}=\mathcal{R}(0, b, s, r)$ est un rectangle de sécurité pour la fonction $G$. On peut donc appliquer la proposition 1 ci-dessus, et il existe une série entière

$$
y(x)=\sum_{m=0}^{\infty} d_{m} x^{m}
$$

de rayon de convergence $R \geqslant s$ et telle que $F(x, y(x))=0$. La fonction $z(x)=\exp (x y(x))$ est analytique pour $|x| \leqslant s$; elle peut donc s'écrire

$$
z(x)=\exp (x y(x))=1+\sum_{m=1}^{\infty} c_{m} x^{m}
$$

et comme $x=1 / n, S_{n}=z(1 / n)$ est bien racine de $f(z)=0$.

Montrons que le rectangle $\mathcal{R}=\mathcal{R}(0, b, s=1 / 25, r=3 / 5)$ est de sécurité pour la fonction $G$, autrement dit montrons que dans ce rectangle, on a $|G(x, y)|<M=20$. Observons d'abord que, pour $t \in \mathbb{C},|t| \leqslant t_{0}$, on a

$$
\left|g^{\prime}(t)\right|=\left|\frac{t}{2}+\frac{t^{2}}{6}+\ldots\right| \leqslant \frac{|t|}{2}+\frac{|t|^{2}}{6}+\ldots \leqslant \frac{t_{0}}{2}+\frac{t_{0}^{2}}{6}+\ldots=g^{\prime}\left(t_{0}\right) .
$$

Il s'ensuit que, pour $(x, y) \in \mathcal{R}$, on a

$$
\left|\frac{\partial F}{\partial x}(x, y)\right|=\left|y g^{\prime}(-x y)\right| \leqslant(|b|+r) g^{\prime}(s(|b|+r)) \leqslant 5.24 .
$$

Nous devons ensuite minorer $\left|\frac{\partial F}{\partial y}\right|$ donné par (41). Il vient

$$
\left|g^{\prime}(y)\right|=\left|\frac{(y-1) e^{y}+1}{y^{2}}\right| \geqslant \frac{|y-1| e^{\Re y}-1}{(|b|+r)^{2}} \geqslant \frac{(|b-1|-r) e^{\Re b-r}-1}{(|b|+r)^{2}} \geqslant 0.427
$$

et il s'ensuit que

$$
\left|\frac{\partial F}{\partial y}(x, y)\right| \geqslant\left|g^{\prime}(y)\right|-\left|x g^{\prime}(-x y)\right| \geqslant\left|g^{\prime}(y)\right|-s g^{\prime}(s(|b|+r))
$$

et par (46), on a

$$
\left|\frac{\partial F}{\partial y}(x, y)\right| \geqslant 0.427-s g^{\prime}(s(|b|+r)) \geqslant 0.427-0.026 \geqslant 0.4 \text {. }
$$


Finalement, de (45) et de (48) on a $|G(x, y)|<5.24 / 0.4<13.02<r / s=15$.

Le point $(x, y(x))$ appartient à ce rectangle de sécurité $\mathcal{R}$, et l'on a donc, pour $|x| \leqslant 0.04,|y(x)| \leqslant|b|+r=8.35$. Par $(43),|z(x)| \leqslant e^{s(|b|+r)} \leqslant 1.4$, et par le théorème des résidus, on a

$$
\left|c_{m}\right|=\left|\int_{|x|=s} \frac{z(x)}{x^{m+1}} d x\right| \leqslant 1.4 / s^{m}=1.4 \times 25^{m} \text {. }
$$

Le calcul des coefficients $c_{m}$ a été fait à l'aide de MAPLE jusqu'à $m=15$. La méthode employée est la suivante : on écrit

$$
y(x)=b+\sum_{m=1}^{\infty} d_{m} x^{m}
$$

avec des coefficients indéterminés. On calcule

$$
e^{y(x)}=e^{b} \exp \left(\sum_{m=1}^{\infty} d_{m} x^{m}\right)=(1+b) \exp \left(\sum_{m=1}^{\infty} d_{m} x^{m}\right),
$$

puis $e^{-x y(x)}$ avec les coefficients indéterminés $d_{m}$, et on identifie pour évaluer les $d_{m}$. On calcule enfin les $c_{m}$ par (43). Cette méthode permet de démontrer par récurrence que $c_{m}$ est un polynôme en $b$ de degré $m$ où $b$ se met en facteur. Un algorithme de calcul plus rapide utilisant la méthode de Newton a été programmé par B. Salvy et a permis d'atteindre $m=32$. La majoration de $\left|c_{m}\right|$ donnée dans (49) n'est sans doute pas très bonne. Le calcul numérique approché des $c_{m}$ jusquà $m=100$ laissent penser que le rayon de convergence est voisin de $1 / 2$. Lorsque l'on remplace la racine $b$ par la racine $z_{k}$ de l'équation $e^{z}=1+z$ (cf. lemme 3 ), le rayon de convergence est proche de $\frac{1}{k+1}$.

5. Les zéros négatifs de $G_{n}$ et $H_{n}$

Soit $n$ impair. K. Dilcher a prouvé dans [4] que le polynôme

$$
H_{n}(z)=\varphi z^{n+1}-(\varphi-1) z^{n}-1
$$

où $\varphi=(1+\sqrt{5}) / 2$ est le nombre d'or, avait un zéro réel négatif $x_{2, n}$. Nous allons montrer

THÉORÈme 4. - Il existe des coefficients réels $c_{1}, c_{2}, \ldots, c_{m}, \ldots$ tels que l'on ait, pour $n$ impair assez grand,

$$
x_{2, n}=-1+\sum_{m=1}^{\infty} \frac{c_{m}}{n^{m}} .
$$


Soit $b=-\frac{\log 5}{2}=-0.805 \ldots$ On $a$

$c_{1}=-b, \quad c_{2}=-\frac{b^{2}}{2}+\frac{b}{2}+\frac{b \sqrt{5}}{10}, \quad c_{3}=-\frac{b^{3}}{6}+\frac{b^{2} \sqrt{5}}{10}+\frac{3 b^{2}}{5}-\frac{3 b}{10}-\frac{b \sqrt{5}}{10}$, et les coefficients $c_{m}$ vérifient $\left|c_{m}\right| \leqslant \frac{5}{4}\left(\frac{11}{2}\right)^{m}$.

DÉMONSTRATION. - Nous allons utiliser le théorème des fonctions implicites analytiques démontré dans le paragraphe 4. On fait d'abord dans (50) le changement de variables $z=-w$. Comme $n$ est impair, l'équation $H_{n}(z)=0$ devient

$$
\varphi w^{n+1}+(\varphi-1) w^{n}-1=0 .
$$

Ensuite, on pose, comme au paragraphe $4, n=1 / x$ et $w=e^{x y}$. On obtient

$$
F(x, y)=\varphi e^{x y}+(\varphi-1)-e^{-y}=0 .
$$

On a $F(0, b)=0$,

$$
\frac{\partial F}{\partial y}(x, y)=\varphi x e^{x y}+e^{-y}
$$

et donc $\frac{\partial F}{\partial y}(0, b)=e^{-b}=\sqrt{5}$. On peut donc appliquer la proposition 1 , et il existe des coefficients $d_{m}$ tels que la série $y(x)=b+\sum_{m=1}^{\infty} d_{m} x^{m}$ vérifie $F(x, y(x))=0$.

On pose $G(x, y)=-\frac{\partial F}{\partial x}(x, y) / \frac{\partial F}{\partial y}(x, y)$. Montrons que le rectangle $\mathcal{R}=$ $\mathcal{R}(0, b, s=2 / 11, r=1 / 3)$ est de sécurité pour l'équation différentielle $y^{\prime}=$ $G(x, y)$. On a

$$
\frac{\partial F}{\partial x}(x, y)=\varphi y e^{x y}
$$

et, pour $|x| \leqslant s$ et $|y-b| \leqslant r$,

$$
\left|\frac{\partial F}{\partial x}(x, y)\right| \leqslant(|b|+r) \varphi e^{s(|b|+r)} \leqslant 2.26
$$

et, $\operatorname{par}(53)$,

$$
\left|\frac{\partial F}{\partial y}(x, y)\right| \geqslant\left|e^{-y}\right|-|x| \varphi e^{|x y|} \geqslant e^{-b-r}-\varphi s e^{s(|b|+r)} \geqslant 1.24 .
$$

On vérifie que $\frac{2.26}{1.24}<\frac{11}{6}$ ce qui assure que $\mathcal{R}$ est bien un rectangle de sécurité. Dans $\mathcal{R}$, on a $|y(x)| \leqslant|b|+r$, et $|z|=|w| \leqslant e^{|x y|} \leqslant e^{s(|b|+r)} \leqslant 5 / 4$, et l'on conclut comme en (49). $\square$

Les calculs numériques de $c_{m}$ et $d_{m}$ laissent prévoir un rayon de convergence voisin de 1 (on a $\sqrt[m]{\left|c_{m}\right|}=1.08 \ldots$ pour $m=125$ et les entiers 
voisins). L'algorithme de calcul de $c_{m}$ et $d_{m}$ permet, comme dans le paragraphe 4 , de montrer que $c_{m}$ et $d_{m}$ sont des polynômes en $b$ de degré $m$ à coefficients dans $\mathbb{Q}(\sqrt{5})$.

La valeur de $b$ a été obtenue comme la racine réelle de $e^{-b}=\sqrt{5} . \mathrm{Si}$ l'on prend pour $b$ une racine complexe, $b=-\frac{\log 5}{2}+2 i k \pi$, on obtiendra le développement en série d'une autre racine de $H_{n}(z)$.

Soit maintenant $n$ pair. K. Dilcher a aussi prouvé dans [4] que le polynôme

$$
G_{n}(z)=(\varphi-1) z^{n+1}-\varphi z^{n}+1
$$

a une racine réelle négative également notée $x_{2, n}$. Le changement de variables $z=-w$ conduit à l'équation

$$
(\varphi-1) w^{n+1}+\varphi w^{n}-1=0
$$

qui est, au signe près, la conjuguée de $(51)$, obtenue en remplaçant $\sqrt{5}$ par $-\sqrt{5}$ dans $(51)$. Le même calcul que précédemment fournit alors le développement en série

$$
x_{2, n}=-1+\sum_{m=1}^{\infty} \frac{\tilde{c}_{m}}{n^{m}} .
$$

où $\tilde{c}_{m}$ est obtenu en remplaçant dans $c_{m}$ le radical $\sqrt{5}$ par $-\sqrt{5}$, ce qui peut se prouver par l'algorithme de calcul des $c_{m}$.

\section{Une série hypergéométrique}

On désignera par $\left[x^{k}\right] y(x)$ le coefficient de $x^{k}$ dans la série entière $y(x)$. En vue de démontrer la proposition 2, nous commençons par deux lemmes.

Lemme 4 (Formule d'inversion de Lagrange, cf. [8], 1.9). - Soit $U(x)$ une série entière avec $U(0) \neq 0$. Posons $W(x)=x / U(x)$. Soit $f(x)$ une série entière; alors la relation $W(f(x))=x$ équivaut à $f(x)=x U(f(x))$ et l'on a pour $n, k \in \mathbb{N}$

$$
n\left[x^{n}\right] f(x)^{k}=k\left[x^{n-k}\right] U(x)^{n} .
$$

En particulier, pour $k=1$ on $a$

$$
\left[x^{n}\right] f(x)=\frac{1}{n}\left[x^{n-1}\right] U(x)^{n} .
$$


LeMme 5 (cf. le lemme 2 de [14]). - Soit une série entière $U(x)=$ $1+\sum_{n \geqslant 1} U_{n} x^{n}$ et $V(x)$ une série entière vérifiant $V(x)=U(x V(x))$. Soit $t \in \mathbb{R}$ et $n \geqslant 1$; si $U_{n}(t)=\left[x^{n}\right] U(x)^{t}$ et $V_{n}(t)=\left[x^{n}\right] V(x)^{t}$, alors

$$
V_{n}(t)=\frac{t}{t+n} U_{n}(t+n) \text {. }
$$

DÉmonstration. - Posons $W(x)=x / U(x)$ et $f(x)=x V(x)$. Il vient alors $W(x V(x))=x$. D'après la formule d'inversion de Lagrange (lemme $4)$, on a pour $n, k \in \mathbb{N}$

$$
\left[x^{n}\right]\left(x^{k} V^{k}(x)\right)=\frac{k}{n}\left[x^{n-k}\right] U(x)^{n},
$$

soit

$$
V_{n}(k)=\left[x^{n+k}\right]\left(x^{k} V^{k}(x)\right)=\frac{k}{n+k}\left[x^{n}\right] U(x)^{n+k}=\frac{k}{n+k} U_{n}(n+k) .
$$

Comme $U_{n}(t)$ et $V_{n}(t)$ sont tous deux des polynômes en $t$ de degré $\leqslant n$, ceci établit l'égalité pour tout nombre réel $t$.

Proposition 2. - Soit $N$ et $P$ deux nombres réels positifs, et $\mu$ un nombre réel. On pose $Q=N-P$. Le rayon de convergence de la série entière

$$
y_{\mu}(x)=\sum_{k \geqslant 0}(-1)^{k} \frac{\mu}{\mu+P k}\left(\begin{array}{c}
(\mu+P k) / N \\
k
\end{array}\right) x^{k}
$$

vaut

$$
R=\frac{N}{\left(P^{P}|Q|^{Q}\right)^{1 / N}}
$$

avec la convention $0^{0}=1$. De plus, pour $|x|<R$, on a $y_{\mu}(x)=\left(y_{1}(x)\right)^{\mu}$ et $y(x)=y_{1}(x)$ vérifie l'équation

$$
y^{N}+x y^{P}-1=0 .
$$

DÉmonstration. - La proposition 2 se trouve dans [1]. Notons que dans [13], 7.32, le cas $N=1$ et $P=2$ est traité comme exemple. La démonstration ci-dessous est due à J. Zeng.

Lorsque $N=P>0$, l'équation (61) devient $y=(1+X)^{-1 / N}$ et l'on retrouve pour $y^{\mu}$ la formule du binôme avec un rayon de convergence égal à 1 . Lorsque $N \neq P$, la valeur de $R$ s'obtient par la formule classique 
de Hadamard $1 / R=\limsup \left(\left[x^{k}\right] y^{\mu}(x)\right)^{1 / k}$ que l'on évalue à l'aide de la formule de Stirling.

D'après le théorème des fonctions implicites analytiques, il existe une série entière $y(x)$ solution de $(61)$ avec $y(0)=1$. On pose $U(x)=(1-x)^{P / N}$ et $V(x)=y^{P}$ vérifie $V(x)=(1-x V(x))^{P / N}$. Comme

$$
U_{k}(t)=\left[x^{k}\right] U(x)^{t}=\left[x^{k}\right](1-x)^{P t / N}=(-1)^{k}\left(\begin{array}{c}
P t / N \\
k
\end{array}\right),
$$

le lemme 5 implique

$$
\left[x^{k}\right] y^{\mu}=\left[x^{k}\right] V(x)^{\mu / P}=(-1)^{k} \frac{\mu}{\mu+P k}\left(\begin{array}{c}
(\mu+P k) / N \\
k
\end{array}\right),
$$

ce qui prouve (59).

THÉORÈmE 5. - Soit $x_{1, n}$ la racine positive et différente de 1 du trinôme $G_{n}$ défini par (3). On a le développement en série, convergent pour $n \geqslant 1$

$$
\begin{aligned}
x_{1, n} & =\varphi^{2}\left(1-\sum_{k=1}^{\infty} \frac{1}{k \varphi^{(2 n+1) k}}\left(\begin{array}{c}
(n+1) k-2 \\
k-1
\end{array}\right)\right) \\
& =\varphi^{2}-\frac{1}{\varphi^{2 n-1}}-\frac{n}{\varphi^{4 n}}-\frac{n(3 n+1)}{2 \varphi^{6 n+1}}-\cdots
\end{aligned}
$$

DÉmONSTRATION. - L'équation $G_{n}(z)=0$ s'écrit aussi

$$
\frac{1}{\varphi} z^{n+1}-\varphi z^{n}+1=0 \text {. }
$$

En effectuant les changements de variables $z=\varphi^{2} y$ et $y=1 / Y$, on obtient

$$
y^{n+1}-y^{n}+\frac{1}{\varphi^{2 n+1}}=0
$$

et

$$
Y-\frac{1}{\varphi^{2 n+1}} Y^{n+1}-1=0 .
$$

On applique à l'équation (63) la proposition 2 , avec $N=1, P=n+1$, $\mu=-1$ et $x=-1 / \varphi^{2 n+1}$, et la formule (59) donne le développement annoncé pour $x_{1, n}$ en remarquant que $z=\varphi^{2} Y^{-1}$ et que

$$
\left(\begin{array}{c}
(n+1) k-1 \\
k
\end{array}\right)=\frac{(n+1) k-1}{k}\left(\begin{array}{c}
(n+1) k-2 \\
k-1
\end{array}\right) .
$$


Par (60), le rayon de convergence vérifie

$$
R=R_{n}=\frac{1}{(n+1)\left(1+\frac{1}{n}\right)^{n}} \geqslant \frac{1}{e(n+1)} .
$$

L'étude de la fonction $t \mapsto \varphi^{2 t+1}-e(t+1)$ montre qu'elle est positive pour $t \geqslant 3 / 2$, ce qui entraîne, par (64), $\frac{1}{\varphi^{2 n+1}}<R_{n}$ pour $n \geqslant 2$ et pour $n=1$, on a, par (64), $R_{1}=1 / 4>1 / \varphi^{3}=0.236 \ldots$, ce qui achève la preuve du théorème 5 .

\section{Bibliographie}

[1] Belardinelli (G.). - Fonctions hypergéométriques de plusieurs variables et résolution analytique des équations algébriques générales. Mémorial des Sciences Math., $\mathrm{n}^{\circ} 145,1960$.

[2] Bieberbach (L.). - Lehrbuch der Funktionentheorie, vol. 1, Elemente der Funktionentheorie, B.G. Teubner, Leipzig und Berlin, 1930.

[3] Demailly (J.-P.). - Analyse numérique et équations différentielles, Presses Universitaires de Grenoble, 1991.

[4] Dilcher (K.). - Zeros of certain cyclotomy-generated polynomials, Fibonacci Quart., 29, 1991, 150-156.

[5] Dilcher (K.), Nulton (J.D.) and Stolarski (K.B.). - The zeros of a certain family of trinomials, Glasgow Math. J., 34, 1992, 55-74.

[6] ERdős (P.) and TURÁN (P.). - On the distribution of the roots of polynomials, Annals of Math. 51, 1950, 105-119.

[7] Feller (W.). - An Introduction to Probability Theory and its Applications, vol. II, 2nd edition, J. Wiley and Sons, 1971.

[8] Henrici (P.). - Applied and computational complex analysis, vol. 1, Power series - integration - conformal mappings - location of zeros, J. Wiley and Sons, 1974.

[9] Hernane (M.-O.). - Localisation des zéros de polynômes intervenant en théorie du signal, Thèse de Magister, Université d'Alger, 1989.

[10] Horadam (A.F.) and Shannon (A.G.). - Cyclotomy-generated Polynomials of Fibonacci Type, In Fibonacci Numbers and Their Applications, 81-97, edited by A.N. Philippou et al., Dordrecht : D. Reidel, 1986.

[11] LaCoume (J.-L.), HaNna (C.) et Nicolas (S.C.). - Etalonnage de l'analyse spectrale par la méthode du modèle autorégressif. Annales de Télécommunications, 36,1981, 579-584.

[12] Nicolas (J.-L.) et Schinzel (A.). - Localisation de zéros de polynômes intervenant en théorie du signal. In Cinquante ans de polynômes, ed. M. Langevin et M. Waldschmidt, Lecture Notes in Math., $\mathrm{n}^{\circ}$ 1415, Springer Verlag, 1990, 167-179.

[13] Whittaker (E.T.) and Watson (G.N.). - A course of modern analysis, 4th ed., Cambridge at the University Press, 1935.

[14] Zeng (J.). - Multinomial convolution polynomials, Discrete Math., 160, 1996, 219228. 